\title{
Synapse specificity of long-term potentiation breaks down at short distances
}

\section{Florian Engert \& Tobias Bonhoeffer}

Nature 388, 279-284 (1997).

Owing to a fault in the final production process, three of the ten panels in the left column of Fig. 3a have been greyed out. This might have conveyed the erroneous impression that three of the ten experiments were conducted differently. The correct figures are reprinted here to emphasize that this is not the case.

a
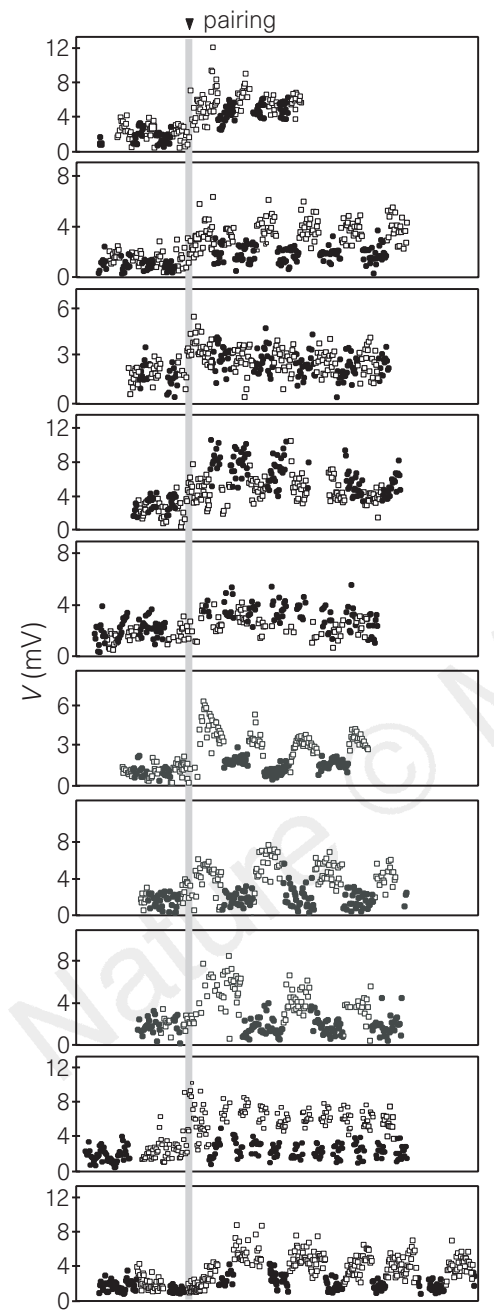

Normalized data
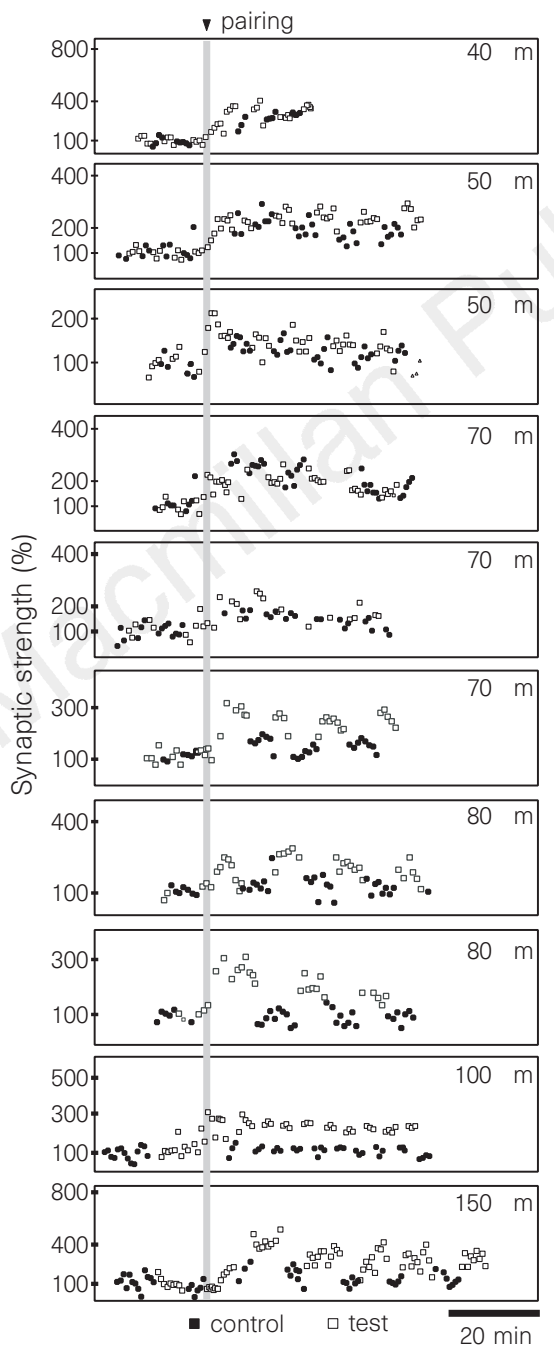
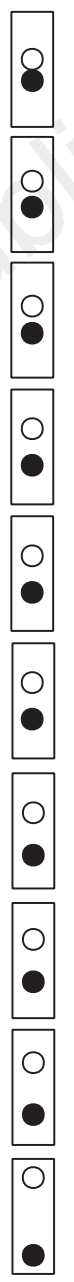

b

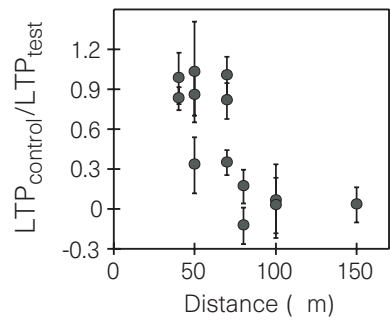


competition and refinement of nerve terminals near the target neuron may thus involve attraction or repulsion of the targetderived factor on the active and inactive nerve terminals, respectively, depending on their cAMP levels.

\section{Methods}

Cell cultures. Cultures of Xenopus spinal neurons were prepared from the neural tube tissue of 1-day-old Xenopus embryos as described ${ }^{12,13}$. The tissue was dissociated in $\mathrm{Ca}^{2+}$ - and $\mathrm{Mg}^{2+}$-free solution $(115 \mathrm{mM} \mathrm{NaCl}, 2.5 \mathrm{mM} \mathrm{KCl}$, $10 \mathrm{mM}$ HEPES, $0.5 \mathrm{mM}$ EDTA, $\mathrm{pH}$ 7.4) for $20 \mathrm{~min}$ and plated on the surface of clean glass coverslips. The culture was preincubated at room temperature (20$\left.22^{\circ} \mathrm{C}\right)$ for $14 \mathrm{~h}$ before use. The culture medium consisted of $49 \%(\mathrm{v} / \mathrm{v})$ Leibovitz medium (GIBCO), 1\% (v/v) fetal bovine serum (HyClone) and $50 \%$ (v/v) Ringer's solution ( $115 \mathrm{mM} \mathrm{NaCl}, 2 \mathrm{mM} \mathrm{CaCl}_{2}, 2.5 \mathrm{mM} \mathrm{KCl}, 10 \mathrm{mM}$ HEPES, pH 7.4). The experiments were carried out in modified Ringer's solution $\left(140 \mathrm{mM} \mathrm{NaCl}, 1 \mathrm{mM} \mathrm{MgCl}_{2}, 1 \mathrm{mM} \mathrm{CaCl}, 5 \mathrm{mM} \mathrm{KCl}, 10 \mathrm{mM}\right.$ HEPES, pH 7.4). Low-Ca ${ }^{2+}(1 \mu \mathrm{M})$ medium was modified Ringer's solution containing $0.9 \mathrm{mM} \mathrm{CaCl}_{2}, 4 \mathrm{mM}$ EDTA.

Production of microscopic chemical gradients. Microscopic gradients of chemicals were produced as described ${ }^{12,13}$. Briefly, repetitive pressure injection of picolitre volumes of solutions containing the chemical was applied through a micropipette that had a tip opening of $\sim 1 \mu \mathrm{m}$. The pressure was applied with an electrically gated pressure application system (Picospritzer General Valve). A standard pressure pulse of 3 p.s.i. in amplitude was applied for $20 \mathrm{~ms}$ to the pipette at a frequency of $2 \mathrm{~Hz}$ using a pulse generator (SD9, Grass Instruments). By measuring the size of droplet in the mineral oil after 50 pulses of repetitive ejection with the same pressure pulse parameters, the average volume of ejected solution per pulse was estimated to be $\sim 1.5 \mathrm{pl}$. Analysis of the gradient ${ }^{12,13}$ indicates that, under these pulse parameters, the average concentration of the chemical was $\sim 10^{3}$-fold lower than that in the pipette, and a concentration gradient of $\sim 5-10 \%$ was created across a growth cone $10 \mu \mathrm{m}$ wide, $100 \mu \mathrm{m}$ away from the tip of the ejecting pipette. The total volume of the saline in the culture dish during the experiment was $5 \mathrm{ml}$. The final average bath concentration of BDNF at the end of the 1-h experiment was $\sim 0.1 \mathrm{ng} \mathrm{ml}^{-1}$.

Measurements of extension and turning of the growth cone. A phasecontrast inverted microscope (Nikon Diaphot) was used to observe the neurite growth. Microscopic images of the neurite were recorded with a CCD camera (Toshiba IK-541RA) attached to microscope and stored on videotape. To find the length of neurite extension, the entire trajectory of the neurite at the end of an hour was measured with a digitizer. To assay growth cone turning, the tip of the pipette containing the chemical was placed $100 \mu \mathrm{m}$ away from the centre of the growth cone and at an angle of $45^{\circ}$ with respect to the direction of initial direction of neurite extension. The direction of the neurite was determined by the last $10-\mu \mathrm{m}$ segment of the neurite. The turning angle was defined by the angle between the original direction of neurite extension and a straight line connecting the positions of the growth cone at the onset and the end of the 1-h period. Only growth cones with a net extension $>5 \mu \mathrm{m}$ over the 1-h period were scored for the turning assay.

Received 30 December 1996; accepted 29 April 1997.

1. Bray, D. \& Hollenbeck, P. J. Growth cone motility and guidance. Annu. Rev. Cell Biol. 4, 43-61 (1988)

2. Keynes, R. \& Cook, G. W. W. Axon guidance molecules. Cell 83, 161-169 (1995).

3. Tessier-Lavigne, M. \& Goodman, C. S. The molecular biology of axon guidance. Science 274, 1123 1133 (1996).

4. Gundersen, R. W. \& Barrett, J. N. Neuronal chemotaxis: chick dorsal root axons turn towards high concentrations of nerve growth factor. Science 206, 1079-1080 (1979).

Bonhoeffer, F. \& Huf, J. Recognition of cell types by axonal growth cones in vitro. Nature 288, 162-164 (1980).

6. Lumsden, A. G. \& Davies, A. M. Earliest sensory nerve fibres are guided to peripheral targets by attractants other than nerve growth factor. Nature 306, 786-788 (1983).

Tessier-Lavigne, M., Placzek, M., Lumsden, A. G., Dodd, J. \& Jessell, T. M. Chemotropic guidance of developing axons in the mammalian central nervous system. Nature 336, 775-778 (1988).

8. Heffner, C. D., Lumsden, A. G. \& O'Leary, D. D. Target control of collateral extension and directional axon growth in the mammalian brain. Science 247, 217-220 (1990).

9. Pini, A. Chemorepulsion of axons in the developing mammalian central nervous system. Science $\mathbf{2 6 1}$, 95-98 (1993).

10. Colamarino, S. A. \& Tessier-Lavigne, M. The axonal chemoattractant netrin-1 is also a chemorepellent for trochlear motor axons. Cell 81, 621-629 (1995).

11. Nakamoto, M. et al. Topographically specific effects of ELF-1 on retinal axon guidance in vitro and retinal axon mapping in vivo. Cell 86, 755-766 (1996).

12. Lohof, A. M., Quillan, M., Dan, Y. \& Poo, M.-m. Asymmetric modulation of cytosolic cAMP activity induces growth cone turning. J. Neurosci. 12, 1253-1261 (1992).

13. Zheng, J. Q., Felder, M., Connor, J. A. \& Poo, M.-m. Turning of nerve growth cones induced by neurotransmitters. Nature 368, 140-144 (1994).
14. Kase, H. et al. K-252 compounds, novel and potent inhibitors of protein kinase C and cyclic nucleotide-dependent protein kinases. Biochem. Biophys. Res. Commun. 142, 436-440 (1987).

15. Kater, S. K., Mattson, M. P., Cohan, C. \& Conner, J. A. Calcium regulation of the neuronal growth cone. Trends Neurosci. 11, 317-323 (1988).

16. Kim, Y.-t. \& Wu, C. F. Reduced growth cone motility in cultured neurons from Drosophila memory mutants with a defective cAMP cascade. J. Neurosci. 16, 5593-5602 (1996).

17. Bixby, J. L. \& Spitzer, N. C. Early differentiation of vertebrate spinal neurons in the absence of voltagedependent $\mathrm{Ca}^{2+}$ and $\mathrm{Na}^{+}$influx. Dev. Biol. 106, 89-96 (1984).

18. Lohof, A. M., Ip, N. Y. \& Poo, M.-m. Potentiation of developing neuromuscular synapses by the neurotrophins NT-3 and BDNF. Nature 363, 350-353 (1993).

19. Rothermel, J. D. \& Parker Botelho, L. H. A mechanistic and kinetic analysis of the interactions of the diastereoisomers of adenosine $3^{\prime}, 5^{\prime}$-(cyclic)phosphorothioate with purified cyclic AMP-dependent protein kinase. J. Biochem. 251, 757-762 (1988).

20. Pin, J.-p. \& Duvoisin, R. The metabotropic glutamate receptors: structure and functions. Neuropharmacology. 34, 1-26 (1995)

21. Meyer-Franke, A., Kaplan, M. R., Pfrieger, F. W. \& Barres, B. A. Characterization of the signaling interactions that promote the survival and growth of developing retinal ganglion cells in culture. Neuron 15, 805-819 (1995).

22. Stoop, R. \& Poo, M.-m. Synaptic modulation by neurotrophic factors: differential and synergistic effects of brain-derived neurotrophic factor and ciliary neurotrophic factor. J. Neurosci. 16, 32563264 (1996).

23. Wayman, G. A. et al. Synergistic activation of the type I adenylyl cyclase by $\mathrm{Ca}^{2+}$ and $\mathrm{G}_{\mathrm{s}}$-coupled receptors in vivo. J. Biol. Chem. 269, 25400-25405 (1994).

24. Yovell, Y., Kandel, E. R., Dudai, Y. \& Abrams, T. W. A quantitative study of the $\mathrm{Ca}^{2+} /$ calmodulin sensitivity of adenylyl cyclase in Aplysia, Drosophila, and rat. J. Neurochem. 59, 1736-1744 (1992).

25. Hamelin, M., Zhou, Y., Su, M.-w., Scott, I. M. \& Culotti, J. G. Expression of the UNC-5 guidance receptor in the touch neurons of $C$. elegans steers their axons dorsally. Nature 364, 327-330 (1993).

26. Wadsworth, W. G., Bhatt, H. \& Hedgecock, E. M. Neuroglia and pioneer neurons express UNC-6 to provide global and local netrin cues for guiding migrations in C. elegans. Neuron 16, 35-46 (1996).

27. Mitchell, K. J. et al. Genetic analysis of netrin genes in Drosophila: Netrins guide CNS commissural axons and peripheral motor axons. Neuron 17, 203-215 (1996).

28. Cooper, J. R., Bloom, F. E. \& Roth, R. H. The Biochemical Basis of Neuropharmacology (7th edn, Oxford University Press, New York, 1996).

29. Laufer, R. \& Changux, J. Calcitonin gene-related peptide elevates cyclic AMP levels in chick skeletal muscle: possible neurotrophic role for a coexisting neuronal messenger. EMBO J. 6, 901-906 (1987).

30. Hempel, C. M., Vincent, P., Adams, S. R., Tsien, R. Y. \& Selverston, A. I. Spatio-temporal dynamics of cyclic AMP signals in an intact neural circuit. Nature 384, 166-169 (1996).

Acknowledgements. This work was supported by a grant from the NIH.

Correspondence and requests for materials should be addressed to M.-m.P. (e-mail: mpoo@ucsd.edu).

\section{Synapse specificity of long- term potentiation breaks down at short distances}

\section{Florian Engert \& Tobias Bonhoeffer}

Max Planck Institute for Psychiatry, Am Klopferspitz 18A,

82152 München-Martinsried, Germany

Long-term potentiation (LTP), the long-lasting increase in synaptic transmission, has been proposed to be a cellular mechanism essential for learning and memory, neuronal development, and circuit reorganization. In the original theoretical ${ }^{1}$ and experimental ${ }^{2}$ work it was assumed that only synapses that had experienced concurrent pre- and postsynaptic activity are subject to synaptic modification. It has since been shown, however, that LTP is also expressed in synapses on neighbouring neurons that have not undergone the induction procedure ${ }^{3-5}$. Yet, it is still believed that this spread of LTP is limited to adjacent postsynaptic cells, and does not occur for synapses on neighbouring input fibres $^{2,6,7}$. However, for technical reasons, tests for input specificity' were always done for synapses relatively far apart. Here we have used a new local superfusion technique, which allowed us to assess the synaptic specificity of LTP with a spatial resolution of $\sim 30 \mu \mathrm{m}$. Our results indicate that there is no input specificity at a distance of less than $70 \mu \mathrm{m}$. Synapses in close proximity to a site of potentiation are also potentiated regardless of their own history of activation, whereas synapses far away show no potentiation.

Synaptic specificity of LTP was examined for synapses between the Schaffer collaterals and the CA1 region in organotypic slice cultures of rat hippocampus ${ }^{3,8}$. By using a local superfusion technique $^{9}$ we localized and individually activated neighbouring groups of synapses with a spatial resolution of less than $30 \mu \mathrm{m}$. 
A pyramidal neuron in the CA1 region of the hippocampus was recorded intracellularly, and an excitatory postsynaptic potential (EPSP) was evoked by stimulation of the Schaffer collaterals (Fig. 1a). Subsequently, transmitter release and hence all EPSPs (and inhibitory postsynaptic potentials) were blocked in the whole culture by replacing the normal extracellular medium with a solution containing a low calcium concentration $(0.8 \mathrm{mM})$ and $10 \mu \mathrm{M}$ cadmium. Synaptic transmission was then restored locally by superfusing a small area of $\sim 30 \mu \mathrm{m}$ diameter (Fig. 1c) with normal medium containing a slightly elevated calcium concentration ( $5 \mathrm{mM}$; see Methods for explanation), no cadmium and a visible dye. The superfusion pipettes were moved along the dendritic arbor of the postsynaptic cell until a group of synapses was found that mediated a small EPSP when the Schaffer-collaterals were stimulated (Fig. 1b).

After one group of active synapses (the 'control' group) was located (Fig. 1d), its vicinity $(40-150 \mu \mathrm{m})$ was searched with the superfusion spot, perpendicular to the Schaffer collaterals, to find another group of synapses (the 'test' group) through which the postsynaptic cell could also be activated (Fig. 1e). LTP was then induced in the test group by pairing postsynaptic depolarization with the presynaptic stimulus (Fig. 1f). Finally, to test whether the control group of synapses was affected by induction of LTP in the neighbouring test group, the superfusion spot was moved back to the control site (Fig. 1g) and these synapses were tested again. Any increment in the control EPSP amplitude would indicate a nonspecific spread of LTP from the test group, as there was no synaptic input activating the control group while the pairing was applied.

To ensure that there was no cross-talk between the two superfusion positions, we performed a control using only the $N$-methylD-aspartate (NMDA) component of the EPSPs. This was done for two reasons: (1) it allowed us to make use of the irreversible openchannel blocker MK-801 (ref. 10), and (2) we could thereby test the component of the EPSP that is presumably responsible for the induction of LTP. NMDA responses were isolated by exchanging the superfusion solution to one containing DNQX $(10 \mu \mathrm{M})$ and no

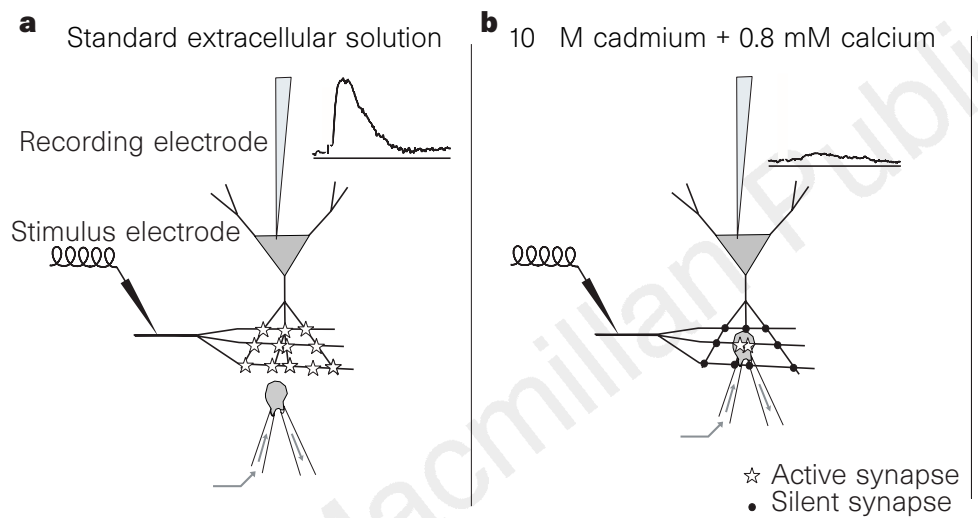

C
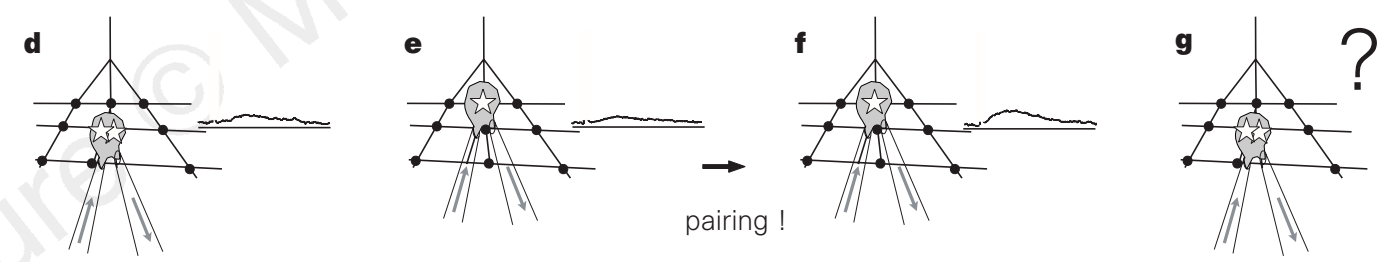

h

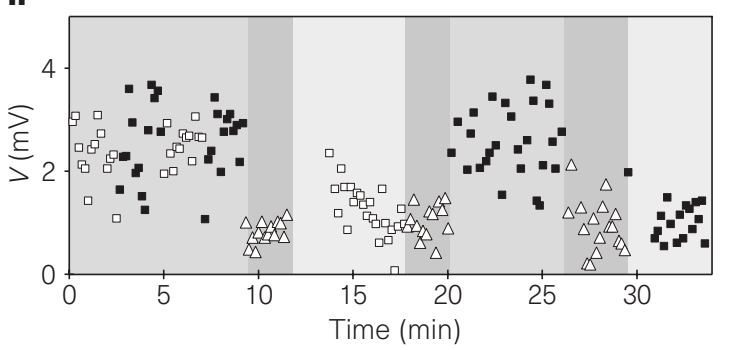

$\triangle$ No spot

$\square$ Superfusion position 1

- Superfusion position 2

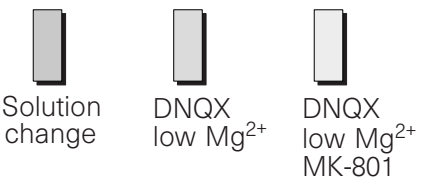

Figure 1 Schematic representation of experimental approach. a, b. Schematic representation of the recording situation. Actual EPSPs are shown as insets in the top right-hand corners. Active synapses are depicted as stars, synapses blocked by $10 \mu \mathrm{M} \mathrm{Cd}^{2+}$ and low $\mathrm{Ca}^{2+}$ as black dots. c, Micrograph of the superfusion spot on the slice culture showing that the size of the spot can be limited to about $30 \mu \mathrm{m}$. The recording electrode can be seen in the top left-hand corner; the application pipette and the shadow of the suction pipette as well as the dyed superfusion solution are seen in the bottom half. $\mathbf{d}-\mathbf{g}$. Sequence of events in an actual experiment ( $\mathbf{d}$ is an enlargement of part of $\mathbf{b}$ ). Further explanations see text. $\mathbf{h}$, the MK-801 test demonstrates that there is no overlap between the two groups of

activated synapses. Different shadings symbolize different solutions in the superfusion pipette. Filled and open squares show responses recorded while superfusion was applied to synapses of group 1 or group 2. After application of MK-801 to group 1 the responses are at noise level (at 16 min; noise level can be judged from the open triangles, which show data collected when the superfusion spot was removed from the culture for purposes of solution change). When the superfusion solution is changed back to medium without MK-801, and is applied to group 2 (20-26 min), responses have not changed compared to the control period (0-9.5 $\mathrm{min})$, indicating that there was no activity in group 2 when group 1 was superfused. 
$\mathrm{Mg}^{2+}$ (Fig. 1h; 0-9.5 min). Synaptic transmission in the nonsuperfused part of the culture was again blocked by $\mathrm{Cd}^{2+}$ and low $\mathrm{Ca}^{2+}$ concentration. We then eliminated the test responses entirely by superfusing these synapses with medium containing MK-801 (Fig. 1h; 12-18 $\mathrm{min}$ ) and went back to the control position, again with no MK-801 in the superfusion, to check whether the responses of these synapses had been affected (Fig. 1h; 20-26 min). There was no change in amplitude, indicating that there was no cross-talk between the test and control synapses; a change in amplitude would have indicated that there was an overlap between the two groups. Even the argument that $\mathrm{Ca}^{2+}$ diffuses differently from MK-801 can be discounted because any extended diffusion of $\mathrm{Ca}^{2+}$ would have prevented us from being able to block the activity fully while superfusing the test position. In none of the control experiments $(n=10$, distances between $40 \mu \mathrm{m}$ and $70 \mu \mathrm{m})$ did we observe a cross-talk between control and test site. Thus it was possible to isolate reliably and activate individually groups of synapses that were $40 \mu \mathrm{m}$ or more apart, allowing us to take a fresh look at input specificity.

Our experiments addressing this issue followed the scheme shown in Fig. 1d-g; the results of our first experiment are shown in Fig. 2a. After establishing a baseline for the two superfusion positions which were separated by $50 \mu \mathrm{m}$ (test and control-50; white and black squares, respectively), the pairing procedure was applied and LTP was induced while synaptic activity was restricted to the test position (note the increasing synaptic responses between 0 and 8 min; white squares). After LTP had been firmly established, the superfusion spot was moved to the control position, and to our surprise, enhancement was also observed for these synapses (Fig. 2a, black squares; 9-12 min and thereafter), despite their lack of activity during the pairing procedure. This was the first demonstration that LTP can spread along the postsynaptic dendrite from activated synapses to nearby synapses that had not undergone the pairing procedure.

To estimate the inter-synaptic distances over which this spreading occurs, we performed recordings where, within a single experiment, the spot was positioned at distances of $50 \mu \mathrm{m}$ and $100 \mu \mathrm{m}$ from the
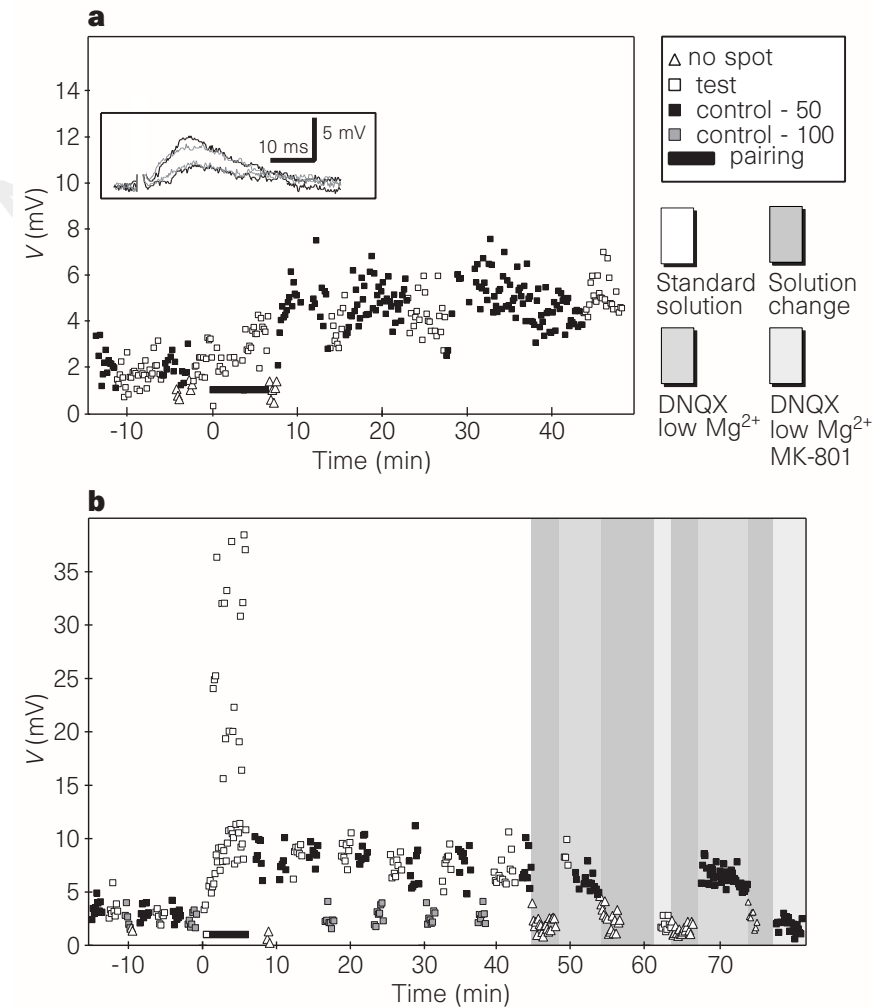

test site. This experiment (Fig. 2b) showed that, although the control synapses at a distance of $50 \mu \mathrm{m}$ (control-50, black squares) were again clearly potentiated, there was no such increase at a distance of $100 \mu \mathrm{m}$ (control-100, grey squares). This demonstrates that the effect has a range of between 50 and $100 \mu \mathrm{m}$, and at the same time proves that we were not observing nonspecific changes, such as changes in general excitability. In this particular experiment (and in five of the eight experiments in which the intersynaptic distance was such that LTP was elicited in both control and test synapses) we also performed the MK-801 control (Fig. 2b; 45-83 min) immediately after the test for input specificity. This control showed that the group of synapses at $50 \mu \mathrm{m}$ could not have experienced any kind of synaptic activity during the induction of LTP in the test group. All of the other experiments of this series are shown in Fig. 3a. It is evident that there is no effect on the control group when the distance between the groups of synapses is greater than $70 \mu \mathrm{m}$, whereas for every single experiment in which it was smaller or equal to this number, the effect was always significant (in all cases $P<0.0001$; Students $t$-test). The ratio of the enhancements for control and test group are plotted against distance for all experiments in Fig. 3b, substantiating our conclusion that the range of the spread of LTP is limited to $\sim 70 \mu \mathrm{m}$ from the site of induction.

To confirm our data using a different experimental approach we performed another series of experiments in which two stimulating electrodes (again termed test and control) were positioned as far apart as possible to ensure that two independent axon bundles were stimulated (Fig. 4a). A slightly larger superfusion spot of $\sim 60 \mu \mathrm{m}$ diameter was used as we were now searching for an area on the dendritic tree with synapses between both stimulated axon bundles and the postsynaptically recorded neuron. After having found such an area we performed 'collision tests' ${ }^{11}$ to verify that the synapses within this confined area were indeed from different and separable inputs. After recording a baseline (with alternating stimulation), postsynaptic depolarization of the recorded neuron was paired only with stimuli of the test electrode, and we examined whether only the test synapses, or also the nearby control synapses, showed enhancement. Also with this experimental approach we found a synaptic

Figure 2 Demonstration that LTP can spread from activated synapses to nearby synapses that have not undergone the pairing procedure. a, After establishing a baseline for the two superfusion positions (test, white squares; control-50, black squares), pairing was applied (black bar) while the synaptic activity was restricted to the test position. After LTP had been established in the test synapses, the superfusion spot was moved to the control-50 position ( $50 \mu \mathrm{m}$ from the test position) and a notable and long-lasting enhancement of the EPSP was also observed for these synapses (black squares, 9-12 min and thereafter). The inset shows typical responses directly before and $\sim 30 \mathrm{~min}$ after the pairing. The thin traces correspond to the white squares (test position), the thick traces to the black squares (control-50 position). b. The same kind of experiment as in a, but a third group (control-100, grey squares) at a distance of $100 \mu \mathrm{m}$ was also examined. Although control-50 was again increased, there is no significant change of EPSP amplitude before or after the pairing procedure (before, $2.4 \pm 0.7 \mathrm{mV}$; after, $2.6 \pm 0.7 \mathrm{mV}$ (mean \pm s.d.) in control-100. The very large responses between 0 and 10 min are caused by action potentials that occurred occasionally during the pairing procedure. In this experiment an MK-801 control (45-83 min) was performed immediately following the actual experiment. 

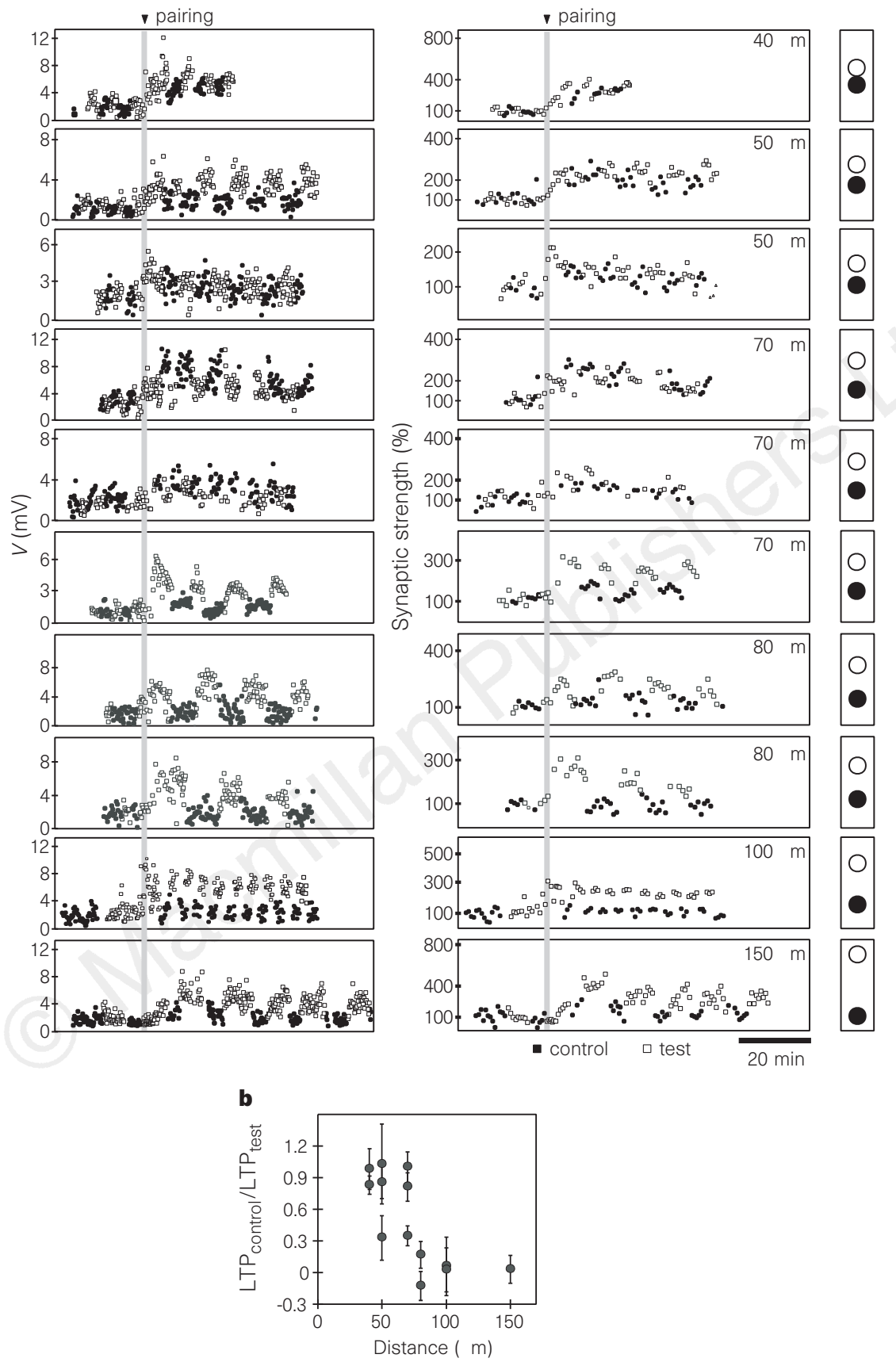

Figure 3 Complete data set for these experiments (excluding those already displayed in Fig. 2). a, Raw data are shown on the left, and data normalized to baseline for test and control group separately, averaged over 5 data points, are shown on the right. The two circles on the extreme right indicate the distance between the test and control group for each experiment. No effect on the control group can be observed when this distance was greater than $70 \mu \mathrm{m}$ (bottom four panels of left and right columns), whereas a significant increase can be seen in all other cases. b. Ratio of the enhancements for control and test group plotted against the distance for all experiments. To obtain a meaningful ratio for the two values we defined, only for the purpose of this figure, no change in synaptic strength (usually 100\%) was 0\% LTP; a doubling was 100\%. Signal size for the calculation of LTP was measured usually 30 min after the beginning of the pairing procedure and averaged over 10 consecutive sweeps. One exception was the cell of the two top panels in $\mathbf{a}$, for which signal amplitude was measured at $25 \mathrm{~min}$. Error bars indicate standard error. 

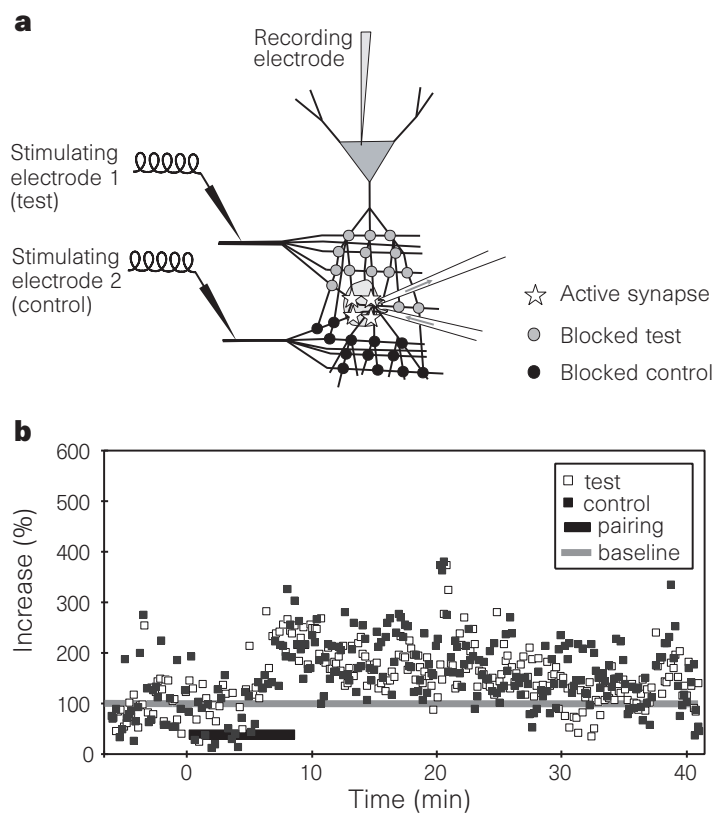

c

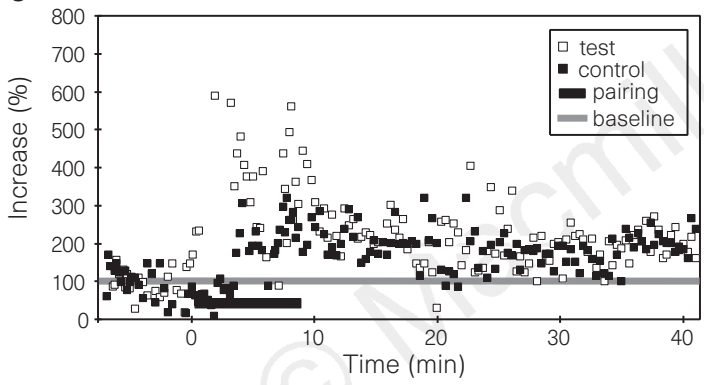

d

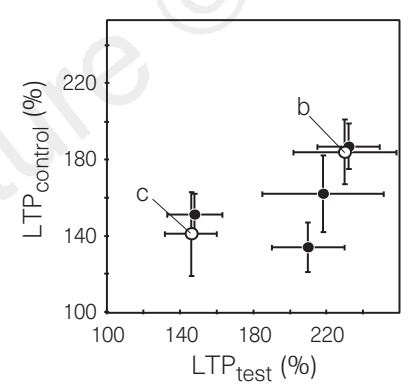

Figure 4 Lack of input specificity shown using a different approach. a, Schematic representation of the experimental approach: two stimulating electrodes were positioned at the two borders of the stratum radiatum and pharmacological isolation was used to isolate an area of $\sim 60 \mu \mathrm{m}$ in diameter that contained synapses between both stimulated fibre bundles and the postsynaptically recorded neuron (active synapses are depicted as white stars, blocked synapses as grey and black dots, for the test and control pathway, respectively). In both experiments shown (b and $\mathbf{c}$ ), increases in the control pathway are of similar size to that in the test pathway. d, Quantitative results for all six such experiments obtained by plotting increase for the control pathways against increase for the test pathways (the experiments of $\mathbf{b}$ and $\mathbf{c}$ are depicted as open circles). Mean and standard error were calculated for 10 sweeps at $30 \mathrm{~min}$. enhancement in the control pathway of similar size to the one in the test pathway (Fig. 4b,c). Sometimes there was a clear delay of onset in the potentiation of the control pathway ( $\sim 5 \mathrm{~min}$ in Fig. $4 \mathrm{c}$; other delays ranged between 0 and $5 \mathrm{~min}$ ), further supporting that the two pathways were independent. The quantitative results for all six such experiments (Fig. 4d) show that, in every experiment, the potentiation in the test pathway was always accompanied by a significant potentiation of the control pathway.

Previous experiments have reported that LTP is input specific. However, owing to the relatively high spatial divergence of the Schaffer collaterals, these experiments had to be performed by stimulating two fairly distant pathways ${ }^{2,6,12}$, and so they did not allow assessment of the specificity of LTP in the range of tens of micrometres. Our method of 'pharmacological isolation' allowed us to tackle this problem in two ways. In the first series of experiments, we could differentiate between synapses that were very close together while being able, by using the MK-801 control, to show that one site was inactive while LTP was induced in the other. Our second series of experiments allowed us, very much in line with earlier studies ${ }^{13}$, to address input specificity by stimulating two pathways. Our specific advantage was that the superfusion technique allowed us to isolate those synapses that are most affected by the spreading of LTP, disregarding the large number of synapses that would, with their irrelevant contribution, otherwise obscure the relatively small effect.

A retrograde messenger has been postulated ${ }^{14-16}$ to mediate between the postsynaptic induction of LTP and its at least partly presynaptic expression ${ }^{17-20}$. Such a messenger would account for the presynaptic spreading of enhancement ${ }^{3-5}$ but, because it was assumed that LTP was input specific, it had to be postulated that the retrograde messenger would only be effective on activated presynaptic terminals ${ }^{14}$. This view has been questioned by reports that presynaptic activity is not necessary, and that artificial postsynaptic elevation of calcium alone is sufficient to induce an LTPlike enhancement $t^{21,22}$. Our results confirm and extend this finding by showing that, also for conventional LTP (as induced by the pairing procedure), presynaptic activity is not a requirement for the expression of LTP. Whereas our first series of experiments demonstrates that presynaptic transmitter release is not required for LTP, the second series shows that not even electrical activity in the presynaptic fibres is necessary to produce synaptic enhancement.

The concept of extracellular ${ }^{7,15,16}$ and intracellular ${ }^{23}$ messengers communicating between neighbouring synapses has recently received increasing attention. We are unable to say conclusively whether the spread of synaptic enhancement reported here is mediated by an extracellular or an intracellular mechanism. However, if an intracellular substance were to mediate the spreading enhancement we would have expected to see occasional failures of this phenomenon in our first series of experiments. These should have occurred if two groups of synapses less than $70 \mu \mathrm{m}$ apart would have come to lie on different dendritic branches, resulting in a 'cytoplasmic distance' considerably greater than $70 \mu \mathrm{m}$. If an intracellular substance were to mediate the spread one might be concerned that a different synaptic morphology in slice cultures could result in less postsynaptic 'compartmentalization', possibly leading to the effect observed here. This seems unlikely, as previous studies in hippocampal ${ }^{24}$ and neocortical ${ }^{25}$ slice cultures have reported the structure of spines, as well as the ratio of shaft to spine synapses, to be largely normal. Moreover, we have examined our cultures electronmicroscopically in that respect (data not shown), and have not observed any abnormalities.

In summary, our two series of experiments demonstrate that input specificity of LTP is not sustained below $70 \mu \mathrm{m}$. However, our data are also compatible with previous reports ${ }^{2,6,12}$ in that they show that enhancement is not totally nonspecific: for distances greater than $70 \mu \mathrm{m}$, synaptic enhancement remains restricted to the 
synapses activated during the induction procedure. Taken together with earlier studies ${ }^{3-5}$, our data suggest that the strict concept of a Hebbian synapse has to be modified to encompass the notion of enhancement spreading several tens of micrometres from the initiation site. If one believes that the Hebbian mechanism is the basis for information storage in the brain then our results indicate that for information storage-not necessarily for information processing-groups of synapses, rather than single synapses, are the basic computational unit.

\section{Methods}

Slice cultures. Organotypic slice cultures of rat hippocampus were prepared ${ }^{8}$ and selected for recording after 2-4 weeks in culture. Monolayered slice cultures were used because the penetration depth of the superfusion technique, which is central to these experiments, is not much more than $\sim 30-50 \mu \mathrm{m}$, so using this technique in acute brain slices is impractical.

Data acquisition and analysis. Intracellular recordings were performed with an AxoClamp-2B amplifier (Axon Instruments) in current-clamp configuration. Sharp electrodes were used to prevent rundown of cells caused by washout of intracellular factors. Data were low-pass filtered at $3 \mathrm{kHz}$, digitally sampled at $5 \mathrm{kHz}$, and stored and analysed with custom-made LabView Software (National Instruments). In the figures the amplitude of the EPSP (in $\mathrm{mV}$ ) is plotted over time. The maximal slopes of EPSPs were also determined in all experiments, but the results are virtually the same (apart from the expected slightly increased scatter of the data points), and so they are not shown.

Electrophysiology. Monopolar stimulus electrodes were made from tungsten wire (TW-8-3, Science Products, Hofheim, Germany), electrolytically sharpened and coated with resin (EPR-4, Clark, Pangbourne, Reading) under microscope control. Recording electrodes were pulled from borosilicate glass and filled with $2 \mathrm{M}$ potassium-methyl-sulphate, resulting in a final resistance of 80-120 M . Modified Hanks balanced salt solution (HBBS) containing $3.2 \mathrm{mM} \mathrm{Ca}^{2+}$ and $0.9 \mathrm{mM} \mathrm{Mg}^{2+}$ was used as the standard extracellular recording solution. To block synaptic transmission, $\mathrm{Ca}^{2+}$ was reduced to $0.8 \mathrm{mM}$ and cadmium $\left(\mathrm{CdCl}_{2}\right)$ in a very mild concentration of $10 \mu \mathrm{M}$ was added (to block voltage-activated calcium channels). Synaptic function was restored locally using a superfusion technique.

LTP and pairing procedure. Only cells with a resting membrane potential below $-65 \mathrm{mV}$ were considered. LTP was verified to be standard in the organotypic cultures by showing that tetanus-induced changes were invariably blocked by the selective agonist AP5 $(50 \mu \mathrm{M}$; data not shown), and that neither postsynaptic depolarization nor presynaptic low-frequency stimuli alone induced LTP (data not shown). The pairing procedure used to induce LTP consisted of $200 \mathrm{~ms}$ postsynaptic current injections of $1.5 \mathrm{nA}$ paired with single presynaptic stimuli applied $5 \mathrm{~ms}$ after start of depolarization. To monitor the change of synaptic strength during the pairing procedure, 30 repetitions at $0.1 \mathrm{~Hz}$ were interleaved with unpaired presynaptic stimuli. For the second series of experiments, stimuli were applied every $5 \mathrm{~s}$, first the paired test stimulus then, consecutively, test and control unpaired (again, 30 repetitions). Superfusion. The superfusion device is described in detail elsewhere? ${ }^{9}$. It consisted of two glass pipettes with tip openings of $\sim 15 \mu \mathrm{m}$ placed opposite each other with an inter-tip distance of $\sim 20 \mu \mathrm{m}$. One pipette served for solution delivery and could be connected to different reservoirs located at a slightly elevated position to provide gravitational pressure to the outflowing solution. Change from one reservoir solution to another was made faster by an additional suction needle in the dead space of the pipette that served to evacuate the interior of the pipettes quickly and enabled the speedy replacement by the solution from the next reservoir. It was therefore possible to use more than one superfusion solution without changing the position of the pipette or the size of the superfused area. The second glass pipette was connected to a peristaltic pump that removed the superfusion solution from the external medium, thereby reducing the affected area to a spot of as little as $30 \mu \mathrm{m}$ in diameter. Size and position of the spot were made visible using food colour in the superfusion solution (see Fig. 1c); the colour has no adverse effects on the electrophysiological properties of the neurons ${ }^{9}$. The two pipettes were mounted on a custom-made set of manipulators to adjust them towards each other before the experiment. These manipulators were themselves mounted on a computer-programmable manipulator (Luigs and Neumann,
Ratingen, Germany), which was used to move the whole arrangement in three dimensions during the recordings. The precision in (re-)positioning the spot was estimated to be $<1 \mu \mathrm{m}$.

In the superfusion solution a $\mathrm{Ca}^{2+}$-concentration of $5 \mathrm{mM}$ was used. It is important to consider that the concentration in the superfusion solution is significantly diluted until it has reached the synaptic terminals deep in the tissue. The actual concentration within the tissue was probably somewhere between 1 and $4 \mathrm{mM}$. Because a minimal concentration of $5 \mathrm{mM}$ in the superfusion solution was necessary to enable synaptic transmission locally (in more than $30 \%$ of cells), the true concentration at the synaptic sites was probably closer to $1 \mathrm{mM}$ than to $4 \mathrm{mM}$. The superfusion onto the synapses in the tissue was rather fast. We found that synaptic potentials could be observed $\sim 30 \mathrm{~s}$ after moving the superfusion spot to the correct position. These potentials vanished $\sim 20 \mathrm{~s}$ after the spot was removed (see Fig. 1h; open triangles show records in which the spot was removed).

MK-801 control. The MK-801 control for overlap between the different groups of synapses was included in five of the eight experiments in which LTP had spread to the control site. It was also done on five cells that did not exhibit LTP in the test group but could conveniently be used to test the confinement of the superfusion spot. To isolate the NMDA component of the EPSP, $10 \mu \mathrm{M}$ DNQX was added to the superfusion solution and $\mathrm{Mg}^{2+}$ was removed. To block the NMDA component irreversibly, $100 \mu \mathrm{M}$ MK-801 (Research Biochemicals Incorporated) was added to the solution.

Received 26 December 1996; accepted 25 April 1997.

1. Hebb, D. O. The Organization of Behavior. A Neuropsychological Theory (Wiley, New York, 1949).

2. Gustafsson, B., Wigström, H., Abraham, W. C. \& Huang, Y.-Y. Long-term potentiation in the hippocampus using depolarizing current pulses as the conditioning stimulus to single volley synaptic potentials. J. Neurosci. 7, 774-780 (1987).

3. Bonhoeffer, T., Staiger, V. \& Aertsen, A. Synaptic plasticity in rat hippocampal slice cultures: local "Hebbian" conjunction of pre- and postsynaptic stimulation leads to distributed synaptic enhancement. Proc. Nat Acad. Sci. USA 86, 8113-8117 (1989).

4. Kossel, A., Bonhoeffer, T. \& Bolz, J. Non-Hebbian synapses in rat visual cortex. NeuroReport 1, 115118 (1990).

5. Schuman, E. M. \& Madison, D. V. Locally distributed synaptic potentiation in the hippocampus. Science 263, 532-536 (1994).

6. Muller, D., Hefft, S. \& Figurov, A. Heterosynaptic interactions between LTP and LTD in CA1 hippocampal slices. Neuron 14, 599-605 (1996).

7. Scanziani, M., Malenka, R. C. \& Nicoll, R. A. Role of intercellular interactions in heterosynaptic longterm depression. Nature 380, 446-450 (1996).

8. Gähwiler, B. H. Morphological differentiation of nerve cells in thin organotypic cultures derived from rat hippocampus and cerebellum. Proc. R. .Soc. Lond. B 211, 287-290 (1981).

9. Veselovsky, N. S., Engert, F. \& Lux, H. D. Fast local superfusion technique. Pflügers Arch. 432, 351-354 (1996).

10. Hessler, N. A., Shirke, A. M. \& Malinow, R. The probability of transmitter release at a mammalian central synapse. Nature 366, 569-572 (1993).

11. Lipski, J. Antidromic activation of neurones as an analytical tool in the study of the central nervous system. J. Neurosci. Methods 4, 1-32 (1981).

12. Durand, G. M., Kovalchuk, Y. \& Konnerth, A. Long-term potentiation and functional synapse induction in developing hippocampus. Nature 381, 71-75 (1996).

13. Otani, S., Connor, J. A. \& Levy, W. B. Long-term potentiation and evidence for novel synaptic association in CA1 stratum oriens of rat hippocampus. Learn. Mem. 2, 101-106 (1995).

14. Williams, J. H., Errington, M. L., Lynch, M. A. \& Bliss, T. V. P. Arachidonic acid induces a long-term activity-dependent enhancement of synaptic transmission in the hippocampus. Nature 341, 739-742 (1989).

15. Schuman, E. M. \& Madison, D. V. A requirement for the intercellular messenger nitric oxide in longterm potentiation. Science 254, 1503-1506 (1991).

16. Arancio, O. et al. Nitric oxide acts directly in the presynaptic neuron to produce long-term potentiaiton in cultured hippocampal neurons. Cells 87, 1025-1035 (1996).

17. Malinow, R. \& Tsien, R. W. Presynaptic enhancement shown by whole-cell recordings of long-term potentiation in hippocampal slices. Nature 346, 177-180 (1990).

18. Larkman, A. U., Hannay, T., Stratford, K. \& Jack, J. J. B. Presynaptic release probability influences the locus of long-term potentiation. Nature 360, 70-73 (1992).

19. Stevens, C. F. \& Wang, Y. Changes in reliability of synaptic function as a mechanism for plasticity. Nature 371, 704-707 (1994).

20. Malgaroli, A. et al. Presynaptic component of long-term potentiation visualized at individual hippocampal synapses. Science 268, 1624-1628 (1995)

21. Malenka, R. C., Kauer, J. A., Zucker, R. S. \& Nicoll, R. A. Postsynaptic calcium is sufficent for potentiation of hippocampal synaptic transmission. Science 242, 81-84 (1988).

22. Neveu, D. \& Zucker, R. S. Long-lasting potentiation and depression without presynaptic activity. J. Neurophysiol. 75, 2157-2160 (1996).

23. Cash, S., Zucker, R. S. \& Poo, M. M. Spread of synaptic depression mediated by presynaptic cytoplasmic signaling. Science 272, 998-1001 (1996).

24. Frotscher, M., Heimrich, B. \& Schwegler, H. Plasticity of identified neurons in slice cultures of hippocampus: a combined Golgi/electron microscopic and immunocytochemical study. Prog. Brain Res. 83, 323-339 (1990)

25. Wolburg, H. \& Bolz, J. Ultrastructural organization of slice cultures from rat visual cortex. J. Neurocytol. 20, 552-563 (1991).

Acknowledgements. We thank M. Häusser, M. Hübener and D. Madison for discussion and comments on the manuscript, and V. Staiger, D. Bühringer and G. Kreutzberg for help with some of the control experiments.

Correspondence and requests for materials should be addressed to T.B. (e-mail: tobi@neuro.mpg.de). 